$11-1-2016$

\title{
An Improved Generalized Estimation Procedure of Current Population Mean in Two-Occasion Successive Sampling
}

G. N. Singh

Indian School of Mines, Dhanbad, India, gnsingh_ism@yahoo.com

Alok Kumar Singh

Indian School of Mines, Dhanbad, India, aksingh.ism@gmail.com

Anup Kumar Sharma

Indian School of Mines, Dhanbad, India, aksharma.ism@gmail.com

Follow this and additional works at: http://digitalcommons.wayne.edu/jmasm

Part of the Applied Statistics Commons, Social and Behavioral Sciences Commons, and the Statistical Theory Commons

\section{Recommended Citation}

Singh, G. N.; Singh, Alok Kumar; and Sharma, Anup Kumar (2016) "An Improved Generalized Estimation Procedure of Current Population Mean in Two-Occasion Successive Sampling," Journal of Modern Applied Statistical Methods: Vol. 15 : Iss. 2 , Article 14. DOI: $10.22237 /$ jmasm/1478002320

Available at: http://digitalcommons.wayne.edu/jmasm/vol15/iss2/14 


\section{An Improved Generalized Estimation Procedure of Current Population Mean in Two-Occasion Successive Sampling}

\section{Cover Page Footnote}

Authors are thankful to the Indian School of Mines, Dhanbad for providing financial and infrastructural support in completion of this research work. 


\section{An Improved Generalized Estimation Procedure of Current Population Mean in Two-Occasion Successive Sampling}

\section{G. N. Singh}

Indian School of Mines

Dhanbad, India

\author{
Alok Kumar Singh \\ Indian School of Mines \\ Dhanbad, India
}

\author{
Anup Kumar Sharma \\ Indian School of Mines \\ Dhanbad, India
}

The present work is an attempt to make use of several auxiliary variables on both occasions for improving the precision of estimates for the current population mean in two-occasion successive sampling. A generalized exponential-cum-regression type estimator of the current population mean is proposed and its optimum replacement strategy has been discussed. Empirical studies are carried out to show the dominance of the proposed estimation procedure over the sample mean estimator and natural successive sampling estimator. Empirical results have been interpreted and suitable recommendations are put forward to survey practitioners.

Keywords: Successive sampling, auxiliary information, bias, mean square error, optimum replacement strategy

\section{Introduction}

There are many problems of practical interest in different fields of the applied and environmental sciences where the various characters of interest have tendencies to change over time. It is often required to monitor the behaviors of such characters at different points of time (occasions) and the patterns of variations occurring over the period of time. For example, an investigator or owner involved in the cold drinks industry may be interested (a) to know the average or total sale of cold drinks in the different seasons, (b) to know the pattern of change in average or total sale of cold drinks in two different seasons, or (c) they may be simultaneously interested to know both (a) and (b). These kinds of problems are well answered by the tools of successive (rotation) sampling.

A. K. Sharma is in the Department of Applied Mathematics. Email at: aksharma.ism@gmail.com. Alok Kumar Singh is a Junior Research Fellow in the Department of Applied Mathematics. Email at: aksingh.ism@gmail.com. G. N. Singh is in the Department of Applied Mathematics._Email at: gnsingh_ism@yahoo.com. 


\section{AN IMPROVED GENERALIZED ESTIMATION PROCEDURE}

The theory of successive (rotation) sampling was initiated by Jessen (1942), where the idea of using the available information gathered on previous occasions during the past surveys was suggested. Jessen (1942) used past information in order to make current estimates more precise in agronomical surveys. This idea was further explored by Patterson (1950), Rao and Graham (1964), Gupta (1979), Das (1982), and Chaturvedi and Tripathi (1983), among others. Sen (1971) extended this theory by utilizing the information on two auxiliary variables, which was available on previous occasions, and suggested estimators of current the population mean in two-occasion successive sampling. Sen $(1972 ; 1973)$ generalized his idea for several auxiliary variables. V. K. Singh, Singh, and Shukla (1991) and G. N. Singh and Singh (2001) used the auxiliary information from the current occasion for estimating the current population mean in two-occasion successive sampling. G. N. Singh (2003) extended this work for h-occasion successive sampling.

In many situations, information on an auxiliary variable may be readily available on the first as well as on the second occasion. For instance, to study the problems related to the public health and welfare of a state or a country, several factors that can be treated as auxiliary variables, such as the number of beds, doctors, and supporting staff in different hospitals, the amount of funds available for medicine, etc. may be known well in advance. Likewise, in other cases, there may be information available on several auxiliary variables and, if efficiently utilized, the estimates could be made more precise.

Utilizing the auxiliary information on both occasions, Feng and Zou (1997), Biradar and Singh (2001), G. N. Singh (2005), G. N. Singh and Priyanka (2006; 2007; 2008; 2010), G. N. Singh and Karna (2009), H. P. Singh and Vishwakarma (2009), G. N. Singh and Prasad (2010), G. N. Singh, Karna, and Prasad (2011), H. P. Singh, Tailor, Singh, and Kim (2011), G. N. Singh and Prasad (2013), and G. N. Singh and Homa (2013) proposed varieties of estimators of the population mean on the current (second) occasions in two-occasion successive sampling.

Motivated with these arguments, the objective of the present work is to propose a more precise estimator of the population mean on the current occasion using the information on $p(p \geq 2)$ stable auxiliary variables which are readily available on both occasions. Utilizing the information on $p$ auxiliary variables, a generalized exponential-cum-regression type estimator of the current population mean in two-occasion successive sampling has been proposed. The dominance of the proposed estimator has been shown over the sample mean and natural successive sampling estimators. Empirical studies have been carried out to justify the proposition of estimator. Results are interpreted, and suitable recommendations have been made. 


\section{SINGH ET AL.}

\section{Formulation of Estimator}

Let $\mathrm{U}=\left(U_{1}, U_{2}, \ldots, U_{N}\right)$ be a finite population of $N$ units which has been sampled over two occasions, and let the character under study be denoted by $x(y)$ on the first (second) occasion. It is assumed that the information on $p$ stable (non-negative integer constant) auxiliary variables $z_{j}(j=1,2, \ldots, p)$, whose population means are known and closely related to $x$ and $y$, are available on the first (second) occasion. Let a simple random sample (without replacement) of size $n$ be drawn on the first occasion. A random subsample of size $m=n \lambda$ is retained (matched) for its use on the second occasion, while a fresh simple random sample (without replacement) of size $u=(n-m)=n \mu$ is drawn on the second occasion from the entire population so that the sample size on the second occasion is also $n$. Here $\lambda$ and $\mu(\lambda+\mu=1)$ are the fractions of the matched and fresh samples, respectively, on the current (second) occasion. The values of $\lambda$ or $\mu$ would be chosen optimally.

The following notations have been considered for use below:

$\bar{X}(\bar{Y})$ : The population mean of the study variable $x(y)$ on the first (second) occasion.

$\bar{Z}_{j}$ : Population mean of the $j^{\text {th }}(j=1,2, \ldots, p)$ auxiliary variable.

$\bar{x}_{n}, \bar{x}_{m}, \bar{y}_{u}, \bar{y}_{m}, \bar{z}_{j n}, \bar{z}_{j u}, \bar{z}_{j m},(j=1,2, \ldots, p)$ : The sample means of the respective variables based on the sample sizes shown in the subscript.

$\rho_{y x}, \rho_{y z_{j}}, \rho_{x z_{j}}, \rho_{z_{j} z_{k}}$ : Population correlation coefficients between the variables shown in the subscript.

$S_{x}^{2}=(N-1)^{-1} \sum_{i=1}^{N}\left(x_{i}-\bar{X}\right)^{2}:$ Population variance of the variable $x$.

$S_{y}^{2}, S_{z_{j}}^{2}$ : Population variances of the variables $y$ and $z_{j}(j=1,2, \ldots, p)$, respectively.

To estimate the population mean $\bar{Y}$ on the current (second) occasion, two independent estimators are suggested. One is a generalized exponential type estimator based on a sample of size $u(=n \mu)$ drawn afresh on the second occasion and given by

$$
T_{u}=\bar{y}_{u} \exp \sum_{j=1}^{p}\left\{\frac{\bar{Z}_{j}-\bar{z}_{j u}}{\bar{Z}_{j}+\bar{z}_{j u}}\right\}
$$




\section{AN IMPROVED GENERALIZED ESTIMATION PROCEDURE}

The second estimator is a generalized exponential-cum-regression type estimator based on the sample of size $m(=n \lambda)$ common to both the occasions and is defined as

$$
T_{m}=\bar{y}_{m}^{*}+\sum_{j=1}^{p} b_{y z_{j}}^{(m)}\left(\bar{Z}_{j}-\bar{z}_{j m}\right)
$$

where

$$
\begin{aligned}
& \bar{y}_{m}^{*}=\bar{y}_{m}+b_{y x}^{(m)}\left(\bar{x}_{n}^{*}-\bar{x}_{m}^{*}\right), \quad \bar{x}_{n}^{*}=\bar{x}_{n} \exp \sum_{j=1}^{p}\left\{\frac{\bar{Z}_{j}-\bar{z}_{j n}}{\bar{Z}_{j}+\bar{z}_{j n}}\right\} \\
& \bar{x}_{m}^{*}=\bar{x}_{m} \exp \sum_{j=1}^{p}\left\{\frac{\bar{Z}_{j}-\bar{z}_{j m}}{\bar{Z}_{j}+\bar{z}_{j m}}\right\}
\end{aligned}
$$

Combining the estimators $T_{u}$ and $T_{m}$, we have the final estimator $T$ of $\bar{Y}$ given as

$$
T=\varphi T_{u}+(1-\varphi) T_{m}
$$

where $\varphi(0 \leq \varphi \leq 1)$ is an unknown constant (scalar) to be determined under certain criterion.

Remark 1: The estimator $T_{u}$ is suitable for estimating the population mean on the current occasion, while the estimator $T_{m}$ is more appropriate for estimating change over two occasions. These two estimators may be derived from the estimator $T$ by choosing $\varphi$ as 1 or 0 , respectively. To handle both problems simultaneously, an optimum choice of $\varphi$ is required.

\section{Properties of the Proposed Estimator}

\section{Bias and Mean Square Error}

Because the estimators $T_{u}$ and $T_{m}$ are generalized exponential and generalized exponential-cum-regression type estimators, they are biased estimators of the population mean $\bar{Y}$. Therefore, the resulting estimator $T$ is also a biased estimator of $\bar{Y}$. The bias $\mathrm{B}($.$) and mean square error \mathrm{M}($.$) of the estimator T$ is derived under 


\section{SINGH ET AL.}

large sample assumption and up to the first order of approximations using the following transformations:

$$
\begin{aligned}
& \bar{y}_{u}=\left(1+e_{1}\right) \bar{Y}, \quad \bar{y}_{m}=\left(1+e_{2}\right) \bar{Y}, \quad \bar{x}_{m}=\left(1+e_{3}\right) \bar{X}, \quad \bar{x}_{n}=\left(1+e_{4}\right) \bar{X}, \\
& \bar{z}_{j u}=\left(1+e_{5 j}\right) \bar{Z}_{j}, \quad \bar{z}_{j m}=\left(1+e_{6 j}\right) \bar{Z}_{j}, \quad \bar{z}_{j n}=\left(1+e_{7 j}\right) \bar{Z}_{j}, \quad s_{y z_{j}}=\left(1+e_{8 j}\right) S_{y z_{j}}, \\
& s_{z_{j}}^{2}(m)=\left(1+e_{9 j}\right) S_{z_{j}}^{2}, \quad s_{x z_{j}}(m)=\left(1+e_{10 j}\right) S_{x z_{j}}, \quad s_{y x}(m)=\left(1+e_{11}\right) S_{y x}, \\
& s_{x}^{2}(m)=\left(1+e_{12}\right) S_{x}^{2}
\end{aligned}
$$

such that $\mathrm{E}\left(e_{i}\right)=0$ and $\mathrm{E}\left(e_{h j}\right)=0,\left|e_{i}\right| \leq 1$ for $i=1,2,3,4,11,12$ and $\left|e_{h j}\right| \leq 1$ for $h=5,6, \ldots, 10, j=1,2,3, \ldots, p$.

Under the above transformations, the estimators $T_{u}$ and $T_{m}$ take the following forms:

$$
\begin{gathered}
T_{u}=\bar{Y}\left(1+e_{1}\right) \exp \sum_{j=1}^{p}\left[-\frac{e_{5 j}}{2}\left(1+\frac{e_{5 j}}{2}\right)^{-1}\right] \\
T_{m}=\bar{Y}\left(1+e_{2}\right)-\frac{\left(1+e_{11}\right)}{\left(1+e_{12}\right)} \beta_{y x} \bar{X}\left(\begin{array}{c}
\left(1+e_{4}\right) \exp \sum_{j=1}^{p} \frac{e_{7 j}}{2}\left(1+\frac{e_{7 j}}{2}\right)^{-1} \\
-\left(1+e_{3}\right) \exp \sum_{j=1}^{p} \frac{e_{6 j}}{2}\left(1+\frac{e_{6 j}}{2}\right)^{-1}
\end{array}\right)
\end{gathered}
$$

Thus, there are the following theorems:

Theorem 1: The bias of the estimator $T$ to the first order of approximations is obtained as

$$
\mathrm{B}(T)=\varphi \mathrm{B}\left(T_{u}\right)+(1-\varphi) \mathrm{B}\left(T_{m}\right)
$$

where

$$
\mathrm{B}\left(T_{u}\right)=\left(\frac{1}{u}-\frac{1}{N}\right) \bar{Y}\left\{\frac{3}{8} \sum_{j=1}^{p} \frac{\alpha_{002}}{\bar{z}_{j}^{2}}+\frac{1}{8} \sum_{j=1}^{p} \frac{\alpha_{002}}{\bar{z}_{j} \bar{z}_{k}}-\frac{1}{2} \sum_{j=1}^{p} \frac{a_{010} \alpha_{001}}{\bar{Y}_{\bar{z}_{j}}}\right\}
$$




\section{AN IMPROVED GENERALIZED ESTIMATION PROCEDURE}

and

$$
\mathrm{B}\left(T_{m}\right)=\left(\frac{1}{m}-\frac{1}{n}\right)\left(\begin{array}{c}
\sum_{j=1}^{p}\left(\frac{1}{2} \frac{\alpha_{100} \alpha_{001}}{\bar{z}_{j}}-\frac{3}{8} \bar{X} \frac{\alpha_{002}}{\bar{z}_{j}^{2}}-\frac{1}{8} \bar{X} \frac{\alpha_{002}}{\bar{z}_{j} \bar{z}_{k}}+\frac{1}{2} \bar{X} \frac{\alpha_{111}}{\bar{z}_{j} S_{y x}}\right) \\
-\sum_{j=1}^{p}\left(\frac{1}{2} \frac{\alpha_{201}}{\bar{z}_{j} S_{x}^{2}}-\frac{\alpha_{012}}{S_{y z_{j}}}+\frac{\alpha_{003}}{S_{z_{j}}^{2}}\right)-\frac{\alpha_{210}}{S_{y x}}+\frac{\alpha_{200}}{S_{x}^{2}}
\end{array}\right)
$$

where $\alpha_{p q r}=\mathrm{E}\left[\left(x_{i}-\bar{X}\right)^{p}\left(y_{i}-\bar{Y}\right)^{q}\left(z_{j}-\bar{Z}_{j}\right)^{r}\right]$ for integers $p, q, r \geq 0$ and $j=1,2, \ldots, p$.

Proof: The bias of the estimator $T$ is given by

$$
\begin{aligned}
\mathrm{B}(T) & =\mathrm{E}[T-\bar{Y}]=\varphi \mathrm{E}\left(T_{u}-\bar{Y}\right)+(1-\varphi) \mathrm{E}\left(T_{m}-\bar{Y}\right) \\
& =\varphi \mathrm{B}\left(T_{u}\right)+(1-\varphi) \mathrm{B}\left(T_{m}\right)
\end{aligned}
$$

where $\mathrm{B}\left(T_{u}\right)=\mathrm{E}\left(T_{u}-\bar{Y}\right)$ and $\mathrm{B}\left(T_{m}\right)=\mathrm{E}\left(T_{m}-\bar{Y}\right)$.

To derive the $\mathrm{B}\left(T_{u}\right)$, proceed as follows:

$$
\mathrm{E}\left(T_{u}-\bar{Y}\right)=\mathrm{E}\left(\bar{Y}\left(1+e_{1}\right) \exp \sum_{j=1}^{p}\left[-\frac{e_{5 j}}{2}\left(1+\frac{e_{5 j}}{2}\right)^{-1}\right]-\bar{Y}\right)
$$

Now, expanding the right hand side of (10) binomially and exponentially and taking expectations and retaining the terms up to the first order of approximations, we have the expression of the bias of the estimator $T_{u}$ as given in (7).

Similarly, the bias of the estimator $T_{m}$ is written as

$$
\begin{aligned}
\mathrm{E}\left(T_{m}-\bar{Y}\right) & \mathrm{E}\left[\bar{Y} e_{2}-\frac{\left(1+e_{11}\right)}{\left(1+e_{12}\right)} \beta_{y x} \bar{X}\left\{\begin{array}{c}
\left(1+e_{4}\right) \exp \left[\sum_{j=1}^{p} \frac{e_{7 j}}{2}\left(1+\frac{e_{7 j}}{2}\right)^{-1}\right] \\
\left.-\left(1+e_{3}\right) \exp \left[\sum_{j=1}^{p} \frac{e_{6 j}}{2}\left(1+\frac{e_{6 j}}{2}\right)^{-1}\right]\right]
\end{array}\right\}\right]
\end{aligned}
$$




\section{SINGH ET AL.}

Expanding (11) binomially and exponentially, taking expectations both sides, and retaining the terms up to the first order of approximations yields the expression of the bias of the estimator $T_{m}$ as shown in (8).

Theorem 2: The mean square error of the estimator $T$ to the first degree of approximation is obtained as

$$
\mathrm{M}(T)=\varphi^{2} \mathrm{M}\left(T_{u}\right)+(1-\varphi)^{2} \mathrm{M}\left(T_{m}\right)+2 \varphi(1-\varphi) \mathrm{C}\left(T_{u}, T_{m}\right)
$$

where

$$
\begin{gathered}
\mathrm{M}\left(T_{u}\right)=\left(\frac{1}{u}-\frac{1}{N}\right) S_{y}^{2}\left[1+\frac{p}{4}-\sum_{j=1}^{p} \rho_{y z_{j}}+\sum_{j \neq k=1}^{p} \rho_{z_{j} z_{k}}\right] \\
\mathrm{M}\left(T_{m}\right)=S_{y}^{2}\left[\left(\frac{1}{m}-\frac{1}{N}\right)\left[\begin{array}{c}
\left.1-\sum_{j=1}^{p}\left(\rho_{y z_{j}}^{2}+\rho_{y z_{j}} \rho_{y z_{k}} \rho_{z_{j} z_{k}}-\rho_{y x} \rho_{y z_{j}}^{2}\right)-2 \rho_{y x}^{2}\right] \\
+\rho_{y x}^{2}\left(1+\frac{p}{4}-\sum_{j=1}^{p} \rho_{y z_{j}}+\sum_{j \neq k=1}^{p} \rho_{z_{j} z_{k}}\right)
\end{array}\right]\right] \\
\mathrm{C}\left(T_{u}, T_{m}\right)=-\frac{1}{N} S_{y}^{2}\left[1-\sum_{j=1}^{p} \rho_{y z_{j}}^{2}\right]
\end{gathered}
$$

Proof: The mean square error of the estimator $T$ is given by

$$
\begin{aligned}
\mathrm{M}(T) & =\mathrm{E}[T-\bar{Y}]^{2}=\mathrm{E}\left[\varphi\left(T_{u}-\bar{Y}\right)+(1-\varphi)\left(T_{m}-\bar{Y}\right)\right]^{2} \\
& =\varphi^{2} \mathrm{M}\left(T_{u}\right)+(1-\varphi)^{2} \mathrm{M}\left(T_{m}\right)+2 \varphi(1-\varphi) \mathrm{C}\left(T_{u}, T_{m}\right)
\end{aligned}
$$

where $\mathrm{C}\left(T_{u}, T_{m}\right)=\mathrm{E}\left[\left(T_{u}-\bar{Y}\right)\left(T_{m}-\bar{Y}\right)\right], \mathrm{M}\left(T_{u}\right)=\mathrm{E}\left(T_{u}-\bar{Y}\right)^{2}, \mathrm{M}\left(T_{m}\right)=\mathrm{E}\left(T_{m}-\bar{Y}\right)^{2}$. To derive the $\mathrm{M}\left(T_{u}\right)$, proceed as follows:

$$
\mathrm{E}\left(T_{u}-\bar{Y}\right)^{2}=\mathrm{E}\left(\bar{Y}\left(1+e_{1}\right) \exp \sum_{j=1}^{p}\left[-\frac{e_{5 j}}{2}\left(1+\frac{e_{5 j}}{2}\right)^{-1}\right]-\bar{Y}\right)^{2}
$$




\section{AN IMPROVED GENERALIZED ESTIMATION PROCEDURE}

Now, expanding the right hand side of (17) binomially and exponentially and taking expectations and retaining the terms up to the first order of approximations, we have the expression of the mean square error of the estimator $T_{u}$ as given in (13).

The mean square error of the estimator $T_{m}$ is written as

$$
\mathrm{E}\left(T_{m}-\bar{Y}\right)^{2}=\mathrm{E}\left[\bar{Y} e_{2}-\frac{\left(1+e_{11}\right)}{\left(1+e_{12}\right)} \beta_{y x} \bar{X}\left\{\begin{array}{c}
\left(1+e_{4}\right) \exp \left[\sum_{j=1}^{p} \frac{e_{7 j}}{2+e_{7 j}}\right] \\
-\left(1+e_{3}\right) \exp \left[\sum_{j=1}^{p} \frac{e_{6 j}}{2+e_{6 j}}\right]
\end{array}\right\}\right]^{2}
$$

Expanding (18) binomially and exponential, taking expectations both sides, and retaining the terms up to the first order of approximations, the expression is derived for the mean square error of the estimator $T_{m}$ as shown in (14). Similarly, the expectation of $\mathrm{C}\left(T_{u}, T_{m}\right)$ may be derived in the form shown in (15).

Remark 2: The above results are derived under the assumption that the coefficients of variation of variables $x, y, z_{j}$, and $z_{k}$ are approximately equal. We have also considered the intuitive assumptions $\rho_{x z_{j}}=\rho_{y z_{j}}(j=1,2,3, \ldots, p)$, as suggested by Cochran (1984) and Feng and Zou (1997). In the light of these assumptions, the expression of $\mathrm{M}\left(T_{m}\right)$ takes the form as shown in (14).

\section{Minimum Mean Square Errors of the Estimator $T$}

Because the mean square error of the estimator $T$ in (12) is a function of the unknown constant (scalar) $\varphi$, it can be minimized with respect to $\varphi$ and, subsequently, the optimum value of $\varphi$ is obtained as

$$
\varphi_{\mathrm{opt}}=\frac{\mathrm{M}\left(T_{m}\right)-\mathrm{C}\left(T_{u}, T_{m}\right)}{\mathrm{M}\left(T_{u}\right)+\mathrm{M}\left(T_{m}\right)-2 \mathrm{C}\left(T_{u}, T_{m}\right)}
$$

From (19), substituting the value of $\varphi_{\text {opt }}$ in (12), we get the optimum mean square error of the estimator $T$ as

$$
\mathrm{M}(T)_{\mathrm{opt}}=\frac{\mathrm{M}\left(T_{u}\right) \mathrm{M}\left(T_{m}\right)-\left\{\mathrm{C}\left(T_{u}, T_{m}\right)\right\}^{2}}{\mathrm{M}\left(T_{u}\right)+\mathrm{M}\left(T_{m}\right)-2 \mathrm{C}\left(T_{u}, T_{m}\right)}
$$




\section{SINGH ET AL.}

Further substituting the values from (13)-(15) into (19) and (20), the simplified values of $\varphi_{\text {opt }}$ and $\mathrm{M}(T)_{\text {opt }}$ are obtained as

$$
\begin{gathered}
\varphi_{\mathrm{opt}}=\frac{\mu\left(A_{9}+\mu A_{8}\right)}{A_{9}-\mu A_{12}+\mu^{2} A_{13}} \\
\mathrm{M}(T)_{\mathrm{opt}}=\left[\frac{A_{18}-\mu^{2} A_{20}-\mu A_{21}}{A_{9}+\mu^{2} A_{13}-\mu A_{12}}\right] \frac{S_{y}^{2}}{n}
\end{gathered}
$$

where

$$
\begin{aligned}
& A_{1}=1-\sum_{j=1}^{p} \rho_{y z_{j}}^{2}+\rho_{y z_{j}} \rho_{y z_{k}} \rho_{z_{j} z_{k}}, \quad A_{2}=\rho_{y x}^{2}\left(1+\frac{p}{4}-\sum_{j=1}^{p} \rho_{y z_{j}}+\frac{1}{4} \sum_{j \neq k=1}^{p} \rho_{z_{j} z_{k}}\right), \\
& A_{3}=\rho_{y x}\left(2 \rho_{y x}-\sum_{j=1}^{p} \rho_{y z_{j}} \rho_{y z_{j}}\right), \quad A_{4}=1-\sum_{j=1}^{p} \rho_{y z_{j}}^{2}, \quad A_{5}=A_{1}+A_{2}-A_{3}, \quad A_{6}=A_{2}-A_{3}, \\
& A_{7}=A_{1}-A_{4}, \quad A_{8}=A_{6}+f A_{7}, \quad A_{9}=A_{5}-A_{8}, \quad A_{10}=1+\frac{p}{4}-\sum_{j=1}^{p} \rho_{y z_{j}}+\frac{1}{4} \sum_{j \neq k=1}^{p} \rho_{z_{j} z_{k}}, \\
& A_{11}=A_{10}-A_{4}, \quad A_{12}=A_{10}-A_{9}+f A_{11}, \quad A_{13}=A_{8}+f A_{11}, \quad A_{14}=A_{10} A_{1}+A_{6} A_{9}, \\
& A_{15}=A_{10} A_{1}, \quad A_{16}=A_{6} A_{10}, \quad A_{17}=A_{4}^{2}-A_{15}, \quad A_{18}=A_{14}-A_{16}-f A_{15}, \\
& A_{19}=A_{14}-A_{15}-A_{16}+f A_{17}, \quad A_{20}=f A_{16}-f^{2} A_{17}, \quad A_{21}=f A_{19}-A_{16}
\end{aligned}
$$

where $f=n / N$.

\section{Optimum Replacement Strategy of the Estimator $T$}

The optimum mean square error $\mathrm{M}(T)_{\mathrm{opt}}$ in (22) is a function on $\mu$, the fraction of the sample to be drawn afresh at the second occasion. It is an important factor in reducing the cost of the survey, therefore, to determine the optimum value of $\mu$ so that $\bar{Y}$ may be estimated with maximum precision and minimum cost. We thus minimize $\mathrm{M}(T)_{\mathrm{opt}}$ with respect to $\mu$ which results in a quadratic equation in $\mu$, which is shown as

$$
\mu^{2} D_{1}-2 \mu D_{2}-D_{3}=0
$$




\section{AN IMPROVED GENERALIZED ESTIMATION PROCEDURE}

where $D_{1}=A_{12} A_{20}+A_{13} A_{21}, D_{2}=A_{10} A_{20}+A_{13} A_{18}, D_{3}=A_{10} A_{21}-A_{12} A_{18}$.

Solving (23) for $\mu$, the solutions of $\mu$ (say $\hat{\mu}$ ) are given as

$$
\hat{\mu}=\frac{D_{2} \pm \sqrt{D_{2}^{2}+D_{1} D_{3}}}{D_{1}}
$$

From (24), it is clear that the real values of $\hat{\mu}$ exist IFF the quantity under the square root is greater than or equal to zero. For any combinations of correlations which satisfy this condition for real solutions, two real values of $\hat{\mu}$ are possible. Hence, while choosing the values of $\hat{\mu}$, it should be remembered that $0 \leq \hat{\mu} \leq 1$, and that all other values of $\hat{\mu}$ are said to be inadmissible. If both the values of $\hat{\mu}$ are admissible, the lowest one is the best choice as it reduces the cost of the survey. From (24), substituting the admissible value of $\hat{\mu}$ (say $\mu_{0}$ ) in (22), we have the optimum value of mean square error of the estimator $T$, which is shown below:

$$
\mathrm{M}(T)_{\mathrm{opt}}^{*}=\left[\frac{A_{18}-\mu_{0}^{2} A_{20}-\mu_{0} A_{21}}{A_{10}+\mu_{0}^{2} A_{13}-\mu_{0} A_{12}}\right] \frac{S_{y}^{2}}{n}
$$

\section{Special Case}

When the $p$ auxiliary variates are mutually uncorrelated, i.e., $\rho_{z_{j} z_{k}}=0$ for $\mathrm{j} \neq k=1,2,3, \ldots, p$, then the expression of the optimum values of $\mu$ and $\mathrm{M}(T)_{\mathrm{opt}}$ reduce to

$$
\hat{\mu}=\frac{D_{2}^{*} \pm \sqrt{D_{2}^{* 2}+D_{1}^{*} D_{3}^{*}}}{D_{1}^{*}}
$$

and

$$
\mathrm{M}(T)_{o p t}^{*}=\left[\frac{A_{18}^{*}-\mu_{0}^{2} A_{20}^{*}-\mu_{0} A_{21}^{*}}{A_{10}^{*}+\mu_{0}^{2} A_{13}^{*}-\mu_{0} A_{12}^{*}}\right] \frac{S_{y}^{2}}{n}
$$

where 


\section{SINGH ET AL.}

$$
\begin{aligned}
& D_{1}^{*}=A_{12}^{*} A_{20}^{*}+A_{13}^{*} A_{21}^{*}, \quad D_{2}^{*}=A_{10}^{*} A_{20}^{*}+A_{13}^{*} A_{18}^{*}, \quad D_{3}^{*}=A_{10}^{*} A_{21}^{*}-A_{12}^{*} A_{18}^{*}, \\
& A_{1}^{*}=1-\sum_{j=1}^{p} \rho_{y z_{j}}^{2}, \quad A_{2}^{*}=\rho_{y x}^{2}\left(1+\frac{p}{4}-\sum_{j=1}^{p} \rho_{y z_{j}}\right), \quad A_{5}^{*}=A_{1}^{*}+A_{2}^{*}-A_{3}, \quad A_{6}^{*}=A_{2}^{*}-A_{3}, \\
& A_{7}^{*}=A_{1}^{*}-A_{4}, \quad A_{8}^{*}=A_{6}^{*}+f A_{7}^{*}, \quad A_{9}^{*}=A_{5}^{*}-A_{8}^{*}, \quad A_{10}^{*}=1+\frac{p}{4}-\sum_{j=1}^{p} \rho_{y z_{j}}, \\
& A_{11}^{*}=A_{10}^{*}-A_{4}, \quad A_{12}^{*}=A_{10}^{*}-A_{9}^{*}+f A_{11}^{*}, \quad A_{13}^{*}=A_{8}^{*}+f A_{11}^{*}, \quad A_{14}^{*}=A_{10}^{*} A_{1}^{*}+A_{6}^{*} A_{9}^{*}, \\
& A_{15}^{*}=A_{10}^{*} A_{1}^{*}, \quad A_{16}^{*}=A_{6}^{*} A_{10}^{*}, \quad A_{17}^{*}=A_{4}^{2}-A_{15}^{*}, \quad A_{18}^{*}=A_{14}^{*}-A_{16}^{*}-f A_{15}^{*}, \\
& A_{19}^{*}=A_{14}^{*}-A_{15}^{*}-A_{16}^{*}+f A_{17}^{*}, \quad A_{20}^{*}=f A_{16}^{*}-f^{2} A_{17}^{*}, \quad A_{21}^{*}=f A_{19}^{*}-A_{16}^{*}
\end{aligned}
$$

\section{Efficiency Comparison}

The percent relative efficiencies of the estimator $T$ with respect to (i) the sample

mean estimator $\bar{y}_{n}$ when there is no matching and (ii) $\hat{\bar{Y}}=\varphi_{u}^{*} \bar{y}_{u}+\left(1-\varphi^{*}\right) \bar{y}_{m}^{\prime}$ when no additional auxiliary information is used at any occasion, where $\bar{y}_{m}^{\prime}=\bar{y}_{m}+\beta_{y x}\left(\bar{x}_{n}-\bar{x}_{m}\right)$, have been obtained for different choices of the correlations involved. Since $\bar{y}_{n}$ and $\hat{\bar{Y}}$ are unbiased estimators of $\bar{Y}$ following Sukhatme, Sukhatme, Sukhatme, and Asok (1984), the variance of $\bar{y}_{n}$ and optimum variance of $\hat{\bar{Y}}$ are given by

$$
\begin{gathered}
\mathrm{V}\left(\bar{y}_{n}\right)=\left(\frac{1}{n}-\frac{1}{N}\right) S_{y}^{2} \\
\mathrm{~V}(\hat{\bar{Y}})_{\mathrm{opt}}=\left[1+\sqrt{1-\rho_{y x}^{2}}\right] \frac{S_{y}^{2}}{2 n}-\frac{S_{y}^{2}}{N}
\end{gathered}
$$

The percent relative efficiencies $E_{1}$ and $E_{2}$ of $T$ (under optimal condition) with respect to $\bar{y}_{n}$ and $\hat{\bar{Y}}$, respectively, are given by

$$
E_{1}=\frac{\mathrm{V}\left(\bar{y}_{n}\right)}{\mathrm{M}(T)_{\mathrm{opt}}^{*}} \times 100, \quad E_{2}=\frac{\mathrm{V}(\hat{\bar{Y}})_{\mathrm{opt}}}{\mathrm{M}(T)_{\mathrm{opt}}^{*}} \times 100
$$




\section{AN IMPROVED GENERALIZED ESTIMATION PROCEDURE}

\section{Empirical Study}

The expression of the optimum $\mu$ (i.e., $\mu_{0}$ ) and the percent relative efficiencies $E_{1}$ and $E_{2}$ are in terms of population correlation coefficients. Therefore, the values of $\mu_{0}, E_{1}$, and $E_{2}$ have been computed for different choices of positive correlations, while the value of $f(=n / N)$ (sampling fraction) is chosen to be 0.1 . For empirical studies, cases of $p=2$ and 3 have been considered.

\section{Case 1}

For $p=2$ and assuming that the two auxiliary variables are correlated, i.e., $\rho_{z_{1} z_{2}} \neq 0$, the values of $A_{1}, A_{2}, A_{3}, A_{4}, A_{9}$, and $A_{10}$ take the form

$$
\begin{aligned}
& A_{1}=1-\left(\rho_{y z_{1}}^{2}+\rho_{y z_{2}}^{2}\right)+2 \rho_{y z_{1}} \rho_{y z_{2}} \rho_{z z_{1} z_{2}}, \quad A_{2}=\rho_{y x}^{2}\left(\frac{3}{2}+2 \rho_{z_{1} z_{2}}-\left(\rho_{y z_{1}}+\rho_{y z_{2}}\right)\right), \\
& A_{3}=\rho_{y x}\left(2 \rho_{y x}-\left(\rho_{y z_{1}}^{2}+\rho_{y z_{2}}^{2}\right)\right), \quad A_{4}=1-\left(\rho_{y z_{1}}^{2}+\rho_{y z_{2}}^{2}\right), \\
& A_{10}=\frac{3}{2}+\frac{1}{2} \rho_{z_{1} z_{2}}-\left(\rho_{y z_{1}}+\rho_{y z_{2}}\right)
\end{aligned}
$$

Substituting these values in (24) and (25) yields the values of optimum $\mathrm{M}(T)_{\mathrm{opt}}^{*}$, $E_{1}$, and $E_{2}$. For different choices of correlations, Tables 1-2 show the optimum values of $\mu$ (i.e., $\left.\mu_{0}\right)$ and percent relative efficiencies $E_{1}$ and $E_{2}$ of the estimator $T$ (under optimal condition) with respect to $\bar{y}_{n}$ and $\hat{\bar{Y}}$, respectively.

\section{Case 2}

For $p=2$ and assuming that the two auxiliary variables are uncorrelated, i.e., $\rho_{z_{1} z_{2}}=0$, the values of $A_{1}^{*}, A_{2}^{*}$, and $A_{10}^{*}$ take the form

$$
A_{1}^{*}=1-\left(\rho_{y z_{1}}^{2}+\rho_{y z_{2}}^{2}\right), \quad A_{2}^{*}=\rho_{y x}^{2}\left(\frac{3}{2}-\left(\rho_{y z_{1}}+\rho_{y z_{2}}\right)\right), \quad A_{10}^{*}=\frac{3}{2}-\left(\rho_{y z_{1}}+\rho_{y z_{2}}\right)
$$

Using these values in (26) and (27), the optimum values of $\mu, E_{1}$, and $E_{2}$ are shown in Table 3. 


\section{SINGH ET AL.}

Table 1. Optimum values of $\mu$ and percent relative efficiencies of $T$ with respect to $\bar{y}_{n}$ and $\hat{\bar{Y}}$ for $\rho_{y x}=0.3$

\begin{tabular}{|c|c|c|c|c|c|c|c|c|c|c|}
\hline \multirow[b]{2}{*}{$\rho_{y z_{2}}$} & \multirow{2}{*}{$\frac{\rho_{y z_{1}}}{\rho_{z_{1} z_{2}}}$} & \multicolumn{3}{|c|}{0.5} & \multicolumn{3}{|c|}{0.6} & \multicolumn{3}{|c|}{0.7} \\
\hline & & $\mu_{0}$ & $E_{1}$ & $E_{2}$ & $\mu_{0}$ & $E_{1}$ & $E_{2}$ & $\mu_{0}$ & $E_{1}$ & $E_{2}$ \\
\hline \multirow[t]{4}{*}{0.4} & 0.3 & 0.7265 & 132.18 & 128.80 & 0.4034 & 168.19 & 163.88 & 0.3533 & 247.90 & 241.56 \\
\hline & 0.4 & 0.7101 & 123.49 & 120.33 & 0.3839 & 152.06 & 148.16 & 0.3872 & 210.05 & 204.67 \\
\hline & 0.5 & 0.7001 & 115.87 & 112.91 & 0.3700 & 138.74 & 135.19 & 0.3360 & 181.76 & 177.10 \\
\hline & 0.6 & 0.6932 & 109.13 & 106.34 & 0.3595 & 127.57 & 124.31 & 0.2952 & 159.92 & 155.82 \\
\hline \multirow[t]{4}{*}{0.6} & 0.3 & 0.6022 & 149.75 & 145.92 & 0.3207 & 193.77 & 188.81 & 0.3411 & 306.04 & 298.21 \\
\hline & 0.4 & 0.5842 & 138.08 & 134.54 & 0.2827 & 170.36 & 166.00 & 0.2607 & 243.37 & 237.15 \\
\hline & 0.5 & 0.5719 & 128.09 & 124.81 & 0.2548 & 151.91 & 148.03 & 0.1979 & 200.80 & 195.67 \\
\hline & 0.6 & 0.5630 & 119.44 & 116.39 & 0.2335 & 137.04 & 133.53 & 0.1475 & 170.24 & 165.88 \\
\hline \multirow[t]{4}{*}{0.8} & 0.3 & 0.5882 & 174.09 & 169.63 & 0.3120 & 234.31 & 228.31 & 0.2669 & 418.57 & 407.85 \\
\hline & 0.4 & 0.5517 & 157.66 & 153.63 & 0.2512 & 197.91 & 192.84 & 0.1760 & 298.60 & 290.96 \\
\hline & 0.5 & 0.5252 & 144.02 & 140.34 & 0.2051 & 170.92 & 166.55 & 0.1034 & 228.96 & 223.10 \\
\hline & 0.6 & 0.5050 & 132.53 & 129.14 & 0.1690 & 150.21 & 146.36 & 0.0441 & 184.02 & 179.31 \\
\hline
\end{tabular}

Table 2. Optimum values of Optimum values of $\mu$ and percent relative efficiencies of $T$ with respect to $\bar{y}_{n}$ and $\hat{\bar{Y}}$ for $\rho_{y x}=0.5$

\begin{tabular}{|c|c|c|c|c|c|c|c|c|c|c|}
\hline \multirow[b]{2}{*}{$\rho_{y z_{2}}$} & \multirow{2}{*}{$\begin{array}{l}\rho_{y z_{1}} \\
\rho_{z_{1} z_{2}}\end{array}$} & \multicolumn{3}{|c|}{0.5} & \multicolumn{3}{|c|}{0.6} & \multicolumn{3}{|c|}{0.7} \\
\hline & & $\mu_{0}$ & $E_{1}$ & $E_{2}$ & $\mu_{0}$ & $E_{1}$ & $E_{2}$ & $\mu_{0}$ & $E_{1}$ & $E_{2}$ \\
\hline \multirow[t]{4}{*}{0.4} & 0.3 & $*$ & -- & -- & 0.3809 & 170.91 & 158.19 & 0.3568 & 249.91 & 231.31 \\
\hline & 0.4 & 0.7440 & 123.96 & 114.73 & 0.3815 & 152.39 & 141.05 & 0.3875 & 208.31 & 192.80 \\
\hline & 0.5 & 0.6739 & 115.31 & 106.73 & 0.3779 & 137.57 & 127.33 & 0.3431 & 178.37 & 165.10 \\
\hline & 0.6 & 0.6411 & 107.74 & 100.72 & 0.3738 & 125.42 & 116.08 & 0.3120 & 155.88 & 144.27 \\
\hline \multirow[t]{4}{*}{0.6} & 0.3 & 0.6748 & 152.04 & 140.72 & 0.2932 & 196.4 & 181.78 & 0.3407 & 307.53 & 284.64 \\
\hline & 0.4 & 0.5913 & 138.47 & 128.16 & 0.2827 & 170.36 & 157.68 & 0.2673 & 240.90 & 222.97 \\
\hline & 0.5 & 0.5579 & 127.09 & 117.63 & 0.2740 & 150.50 & 139.29 & 0.2192 & 197.38 & 182.69 \\
\hline & 0.6 & 0.5386 & 117.45 & 108.71 & 0.2669 & 134.83 & 124.80 & 0.1851 & 166.90 & 154.48 \\
\hline \multirow[t]{4}{*}{0.8} & 0.3 & 0.6209 & 176.39 & 163.26 & 0.3015 & 236.81 & 219.19 & 0.2670 & 418.02 & 386.91 \\
\hline & 0.4 & 0.5506 & 157.54 & 145.81 & 0.2567 & 197.19 & 182.51 & 0.1865 & 294.69 & 272.76 \\
\hline & 0.5 & 0.5134 & 142.28 & 131.69 & 0.2285 & 168.91 & 156.34 & 0.1319 & 225.58 & 208.79 \\
\hline & 0.6 & 0.4896 & 129.72 & 120.07 & 0.2089 & 147.72 & 136.73 & 0.0924 & 181.87 & 168.33 \\
\hline
\end{tabular}

Note: "*” indicates $\mu_{0}$ does not exists and "--" indicates no gain. 


\section{AN IMPROVED GENERALIZED ESTIMATION PROCEDURE}

Table 3. Optimum values of Optimum values of $\mu$ and percent relative efficiencies of $T$ with respect to $\bar{y}_{n}$ and $\hat{\bar{Y}}$ for $\rho_{z_{1} z_{2}}=0.0$

\begin{tabular}{|c|c|c|c|c|c|c|c|c|c|c|}
\hline \multirow[b]{2}{*}{$\rho_{y x}$} & \multirow{2}{*}{$\begin{array}{l}\rho_{y z_{1}} \\
\rho_{y z_{2}}\end{array}$} & \multicolumn{3}{|c|}{0.5} & \multicolumn{3}{|c|}{0.6} & \multicolumn{3}{|c|}{0.7} \\
\hline & & $\mu_{0}$ & $E_{1}$ & $E_{2}$ & $\mu_{0}$ & $E_{1}$ & $E_{2}$ & $\mu_{0}$ & $E_{1}$ & $E_{2}$ \\
\hline \multirow[t]{4}{*}{0.5} & 0.3 & 0.4203 & 138.95 & 128.61 & 0.4317 & 158.35 & 146.57 & 0.3727 & 187.50 & 173.54 \\
\hline & 0.4 & 0.4874 & 156.18 & 144.55 & 0.5084 & 181.27 & 167.78 & 0.4661 & 219.80 & 203.44 \\
\hline & 0.5 & 0.5084 & 181.27 & 167.78 & 0.5359 & 216.08 & 200.00 & 0.4938 & 272.88 & 252.57 \\
\hline & 0.6 & 0.4661 & 219.80 & 203.44 & 0.4938 & 272.88 & 252.57 & 0.4112 & 371.39 & 343.75 \\
\hline \multirow[t]{4}{*}{0.7} & 0.3 & 0.5632 & 157.93 & 132.85 & 0.5865 & 185.15 & 155.75 & 0.6036 & 228.38 & 192.11 \\
\hline & 0.4 & 0.6015 & 182.74 & 153.71 & 0.6312 & 220.87 & 185.79 & 0.6662 & 287.47 & 241.82 \\
\hline & 0.5 & 0.6312 & 220.87 & 185.79 & 0.6759 & 281.34 & 236.66 & 0.7712 & 417.32 & 351.04 \\
\hline & 0.6 & 0.6662 & 287.47 & 241.82 & 0.7712 & 417.32 & 351.04 & * & -- & -- \\
\hline \multirow[t]{4}{*}{0.9} & 0.3 & 0.9388 & 268.34 & 184.24 & * & -- & -- & * & -- & -- \\
\hline & 0.4 & * & -- & -- & * & -- & -- & * & -- & -- \\
\hline & 0.5 & * & -- & -- & * & -- & -- & * & -- & -- \\
\hline & 0.6 & * & -- & -- & * & -- & -- & * & -- & -- \\
\hline
\end{tabular}

Note: "*” indicates $\mu_{0}$ does not exists and "--“ indicates no gain.

\section{Case 3}

For $p=3$ and assuming that the two auxiliary variables are correlated, i.e., $\rho_{z_{j} z_{k}} \neq 0$ for $j \neq k=1,2,3$, the values of $A_{1}, A_{2}, A_{3}, A_{4}$, and $A_{10}$ take the form

$$
\begin{aligned}
& A_{1}=1-\left(\rho_{y z_{1}}^{2}+\rho_{y z_{2}}^{2}+\rho_{y z_{3}}^{2}\right)+2\left(\rho_{y z_{1}} \rho_{y z_{2}} \rho_{z_{1} z_{2}}+\rho_{y z_{1}} \rho_{y z_{3}} \rho_{z_{1} z_{3}}+\rho_{y z_{2}} \rho_{y z_{3}} \rho_{z_{2} z_{3}}\right), \\
& A_{2}=\rho_{y x}^{2}\left(\frac{7}{4}+2\left(\rho_{z_{1} z_{2}}+\rho_{z_{1} z_{3}}+\rho_{z_{2} z_{3}}\right)-\left(\rho_{y z_{1}}+\rho_{y z_{2}}+\rho_{y z_{3}}\right)\right), \\
& A_{3}=\rho_{y x}\left(2 \rho_{y x}-\left(\rho_{y z_{1}}^{2}+\rho_{y z_{2}}^{2}+\rho_{y z_{3}}^{2}\right)\right), \quad A_{4}=1-\left(\rho_{y z_{1}}^{2}+\rho_{y z_{2}}^{2}+\rho_{y z_{3}}^{2}\right), \\
& A_{10}=\frac{7}{4}+\frac{1}{2}\left(\rho_{z_{1} z_{2}}+\rho_{z_{1} z_{3}}+\rho_{z_{2} z_{3}}\right)-\left(\rho_{y z_{1}}+\rho_{y z_{2}}+\rho_{y z_{3}}\right)
\end{aligned}
$$

In this case there are seven different correlations. For a few sets of these seven correlations, the optimum value of $\mu$ (i.e., $\mu_{0}$ ) and percent relative efficiencies $E_{1}$ and $E_{2}$ of the estimator $T$ (under optimal condition) with respect to $\bar{y}_{n}$ and $\hat{\bar{Y}}$ have been computed and shown below: 


\section{SINGH ET AL.}

Set 1:

$$
\begin{aligned}
& \rho_{y x}=0.3, \quad \rho_{y z_{1}}=0.5, \quad \rho_{y z_{2}}=0.6, \quad \rho_{y z_{3}}=0.5, \quad \rho_{z_{1} z_{2}}=0.3, \quad \rho_{z_{1} z_{3}}=0.4, \\
& \rho_{z_{2} z_{3}}=0.6, \quad \mu_{0}=0.3664, \quad E_{1}=104.37, \quad E_{2}=101.37
\end{aligned}
$$

Set 2:

$$
\begin{aligned}
& \rho_{y x}=0.3, \quad \rho_{y z_{1}}=0.6, \quad \rho_{y z_{2}}=0.6, \quad \rho_{y z_{3}}=0.5, \quad \rho_{z_{1} z_{2}}=0.3, \quad \rho_{z_{1} z_{3}}=0.4, \\
& \rho_{z_{2} z_{3}}=0.6, \quad \mu_{0}=0.2900, \quad E_{1}=110.60, \quad E_{2}=107.77
\end{aligned}
$$

Set 3:

$$
\begin{aligned}
& \rho_{y x}=0.3, \quad \rho_{y z_{1}}=0.7, \quad \rho_{y z_{2}}=0.6, \quad \rho_{y z_{3}}=0.5, \quad \rho_{z_{1} z_{2}}=0.3, \quad \rho_{z_{1} z_{3}}=0.4, \\
& \rho_{z_{2} z_{3}}=0.6, \quad \mu_{0}=0.2393, \quad E_{1}=119.33, \quad E_{2}=116.27
\end{aligned}
$$

\section{Set 4:}

$$
\begin{aligned}
& \rho_{y x}=0.3, \quad \rho_{y z_{1}}=0.8, \quad \rho_{y z_{2}}=0.6, \quad \rho_{y z_{3}}=0.5, \quad \rho_{z_{1} z_{2}}=0.3, \quad \rho_{z_{1} z_{3}}=0.4, \\
& \rho_{z_{2} z_{3}}=0.6, \quad \mu_{0}=0.2105, \quad E_{1}=131.59, \quad E_{2}=128.22
\end{aligned}
$$

\section{Case 4}

For $p=3$ and assuming that the two auxiliary variables are uncorrelated, i.e., $\rho_{z_{1} z_{2}}=0$ for $j \neq k=1,2,3$, the values of $A_{1}, A_{2}$, and $A_{10}$ take the form

$$
\begin{aligned}
& A_{1}=1-\left(\rho_{y z_{1}}^{2}+\rho_{y z_{2}}^{2}+\rho_{y z_{3}}^{2}\right), \quad A_{2}=\rho_{y x}^{2}\left(\frac{7}{4}-\left(\rho_{y z_{1}}+\rho_{y z_{2}}+\rho_{y z_{3}}\right)\right), \\
& A_{10}=\frac{7}{4}-\left(\rho_{y z_{1}}+\rho_{y z_{2}}+\rho_{y z_{3}}\right)
\end{aligned}
$$

For a few sets of the above four correlations, the values of the optimum value of $\mu$ (i.e., $\left.\mu_{0}\right)$ and percent relative efficiencies $E_{1}$ and $E_{2}$ are shown below:

\section{Set 1:}

$$
\begin{aligned}
& \rho_{y x}=0.3, \quad \rho_{y z_{1}}=0.5, \quad \rho_{y z_{2}}=0.6, \quad \rho_{y z_{3}}=0.4, \quad \mu_{0}=0.6004, \quad E_{1}=382.42, \\
& E_{2}=372.64
\end{aligned}
$$




\section{AN IMPROVED GENERALIZED ESTIMATION PROCEDURE}

Set 2:

$$
\begin{aligned}
& \rho_{y x}=0.4, \quad \rho_{y z_{1}}=0.5, \quad \rho_{y z_{2}}=0.6, \quad \rho_{y z_{3}}=0.4, \quad \mu_{0}=0.7981, \quad E_{1}=397.89, \\
& E_{2}=379.44
\end{aligned}
$$

Set 3:

$$
\begin{aligned}
& \rho_{y x}=0.5, \quad \rho_{y z_{1}}=0.5, \quad \rho_{y z_{2}}=0.6, \quad \rho_{y z_{3}}=0.4, \quad \mu_{0}=0.3807, \quad E_{1}=449.12, \\
& E_{2}=415.67
\end{aligned}
$$

\section{Set 4:}

$$
\begin{aligned}
& \rho_{y x}=0.6, \quad \rho_{y z_{1}}=0.5, \quad \rho_{y z_{2}}=0.6, \quad \rho_{y z_{3}}=0.4, \quad \mu_{0}=0.6317, \quad E_{1}=568.19, \\
& E_{2}=505.06
\end{aligned}
$$

\section{Conclusion}

1. From Tables 1-2 it is vindicated that:

a. For the fixed values of $\rho_{y x}, \rho_{z_{1} z_{2}}$, and $\rho_{y z_{1}}$, the values of $\mu_{0}$ decrease and $E_{1}$ and $E_{2}$ increase with the increasing values of $\rho_{y z_{2}}$. Similarly, for fixed values of $\rho_{y x}, \rho_{z_{1} z_{2}}$, and $\rho_{y z_{2}}$, the optimum value of $\mu_{0}$ decrease and $E_{1}$ and $E_{2}$ increase with the increasing values of $\rho_{y z_{1}}$. These patterns indicate that a smaller fresh sample on the current occasion is required if highly correlated auxiliary variables are available.

b. For the fixed values of $\rho_{y x}, \rho_{y z_{1}}$, and $\rho_{y z_{2}}$, the values of $\mu_{0}, E_{1}$, and $E_{2}$ decrease with the increasing values of $\rho_{z_{1} z_{2}}$; this means that the auxiliary variables are quite sensitive with respect to the relation between them.

2. From Table 3, i.e., when the auxiliary variables are uncorrelated, it has been observed that

a. For fixed values of $\rho_{y z_{1}}$ and $\rho_{y z_{2}}$, the values of $E_{1}$ and $E_{2}$ increase with increasing value of $\rho_{y x}$, while no definite patterns are observed in $\mu_{0}$. 


\section{SINGH ET AL.}

b. For fixed values of $\rho_{y x}$ and $\rho_{y z_{1}}$, the values of $E_{1}$ and $E_{2}$ increase with increasing value of $\rho_{y z_{2}}$, while no definite patterns are observed in $\mu_{0}$. Similar patterns are visible for the case when the values of $\rho_{y x}$ and $\rho_{y z_{2}}$ are fixed and increasing values of $\rho_{y z_{1}}$ are observed.

3. For $p=3$ and when the three auxiliary variables are uncorrelated, for fixed values of $\rho_{y x}, \rho_{z_{1} z_{2}}, \rho_{z_{2} z_{3}}, \rho_{z_{1} z_{3}}, \rho_{y z_{2}}$, and $\rho_{y z_{3}}$, the values of $\mu_{0}$ decrease while $E_{1}$ and $E_{2}$ increase with the increasing values of $\rho_{y z_{1}}$. Similar patterns are observed if the case for the increasing values of $\rho_{y z_{2}}$ or $\rho_{y z_{3}}$ is taken into account.

4. For $p=3$ and when the three auxiliary variables are mutually correlated, we observed that no specific pattern is seen as for so many combinations of correlations the optimum values of $\mu_{0}$ do not exist. This behavior suggests that the correlation between the auxiliary variable do not play a significant role in terms of the proposed estimator.

5. It could be seen that the results are more appreciable for one and two auxiliary variables, while when the number of auxiliary variables increases, the expressions become complex due to the increase in the number of correlations. Hence, practically, it is more realistic to use two auxiliary variables out of several available auxiliary variables.

Thus, it is clear that the use of the auxiliary variables is highly rewarding in terms of the proposed estimator. It is also clear that, if the information on highly correlated auxiliary variables is used, only a relatively small fraction of the sample on the current (second) occasion is desired to be replaced by a fresh sample, which reduces the cost of the survey. Hence, it can be recommended for future use.

\section{References}

Biradar, R. S., \& Singh, H. P. (2001). Successive sampling using auxiliary information on both occasions. Bulletin of the Calcutta Statistical Association, 51(203-204), 243-251.

Chaturvedi, D. K., \& Tripathi, T. P. (1983). Estimation of population ratio on two occasions using multivariate auxiliary information. Journal of the Indian Statistical Association, 21, 113-120. 


\section{AN IMPROVED GENERALIZED ESTIMATION PROCEDURE}

Cochran, W. G. (1984). Sampling techniques (3rd ed.). New Delhi, India: Wiley Eastern.

Das, A. K. (1982). Estimation of population ratio on two occasions. Journal of the Indian Society of Agricultural Statistics, 34(2), 1-9.

Feng, S., \& Zou, G. (1997). Sample rotation method with auxiliary variable. Communications in Statistics - Theory and Methods, 26(6), 1497-1509. doi: 10.1080/03610929708831996

Gupta, P. C. (1979). Sampling on two successive occasions. Journal of Statistical Research, 13(13), 7-16.

Jessen, R. J. (1942). Statistical Investigation of a Sample Survey for obtaining farm facts. Agricultural Research Bulletins, 304, 1-104. Retrieved from http://lib.dr.iastate.edu/ag_researchbulletins/27/

Patterson, H. D. (1950). Sampling on successive occasions with partial replacement of units. Journal of the Royal Statistical Society. Series B (Methodological), 12(2), 241-255. Available from http://www.jstor.org/stable/2983982

Rao, J. N. K., \& Graham, J. E. (1964). Rotation design for sampling on repeated occasions. Journal of the American Statistical Association, 59(306), 492509. doi: 10.1080/01621459.1964.10482175

Sen, A. R. (1971). Successive sampling with two auxiliary variables. Sankhyā: The Indian Journal of Statistics, Series B, 33(3/4), 371-378. Available from http://www.jstor.org/stable/25051764

Sen, A. R. (1972). Successive sampling with $p(p \geq 1)$ auxiliary variables. The Annals of Mathematical Statistics, 43(6), 2031-2034. Available from http://www.jstor.org/stable/2240218

Sen, A. R. (1973). Note: Theory and application of sampling on repeated occasions with several auxiliary variables. Biometrics, 29(2), 381-385. doi: $10.2307 / 2529401$

Singh, G. N. (2003). Estimation of population mean using auxiliary information on recent occasion in h-occasion successive sampling. Statistics in Transition, 6(4), 523-532.

Singh, G. N. (2005). On the use of chain-type ratio estimator in successive sampling. Statistics in Transition, 7(1), 21-26.

Singh, G. N., \& Homa, F. (2013). Effective rotation patterns in successive sampling over two occasions. Journal of Statistical Theory and Practice, 7(1), 146-155. doi: 10.1080/15598608.2012.755484 


\section{SINGH ET AL.}

Singh, G. N., \& Karna, J. P. (2009). Estimation of population mean on current occasions in two-occasion successive sampling. Metron, 67(1), 69-85.

Singh, G. N., Karna, J. P., \& Prasad, S. (2011). On the use of multiple auxiliary variables in estimation of current population mean in two-occasion successive sampling. Sri Lankan Journal of Applied Statistics, 12, 101-116. doi: 10.4038/sljastats.v12i0.4970

Singh, G. N., \& Prasad, S. (2010). Some estimator of population mean in two-occasion rotation patterns. Association for the Advancement of Modelling \& Simulation Techniques in Enterprises, 47(2), 1-18.

Singh, G. N., \& Prasad, S. (2013). Best linear unbiased estimators of population mean on current occasion in two-occasion successive sampling. Statistics in Transition - New Series, 14(1), 57-74.

Singh, G. N., \& Priyanka, K. (2006). On the use of chain-type ratio to difference estimator in successive sampling. International Journal of Applied Mathematics and Statistics, 5(S06), 41-49.

Singh, G. N., \& Priyanka, K. (2007). On the use of auxiliary information in search of good rotation patterns on successive occasions. International Journal of Statistics and Economics, 1(A07), 42-60.

Singh, G. N., \& Priyanka, K. (2008). On the use of several auxiliary variates to improve the precision of estimates at current occasion. Journal of the Indian Society of Agricultural Statistics, 62(3), 253-265. Retrieved from http://isas.org.in/jisas/jsp/volume/vol62/7-G.N.Singh.pdf

Singh, G. N., \& Priyanka, K. (2010). Estimation of population mean at current occasion in presence of several varying auxiliary variates in two-occasion successive sampling. Statistics in Transition - New Series, 11(1), 105-126.

Singh, G. N., \& Singh, V. K. (2001). On the use of auxiliary information in successive sampling. Journal of the Indian Society of Agricultural Statistics, 54(1), 1-12. Retrieved from http://www.isas.org.in/jsp/volume/vol54/G.N.\%20Singh\%20.pdf

Singh, H. P., Tailor, R., Singh, S., \& Kim, J.-M., (2011). Estimation of population variance in successive sampling. Quality \& Quantity, 45(3), 477-494. doi: 10.1007/s11135-009-9309-9

Singh, H. P., \& Vishwakarma, G. K. (2009). A general procedure for estimating population mean in successive sampling. Communications in Statistics - Theory and Methods, 38(2), 293-308. doi: 10.1080/03610920802169594 


\section{AN IMPROVED GENERALIZED ESTIMATION PROCEDURE}

Singh, V. K., Singh, G. N., \& Shukla, D. (1991). An efficient family of ratio-cum-difference type estimators in successive sampling over two occasions. Journal of Scientific Research, 41(C), 149-159.

Sukhatme, P. V., Sukhatme, B. V., Sukhatme, S., \& Asok, C. (1984). Sampling theory of surveys with applications. Ames, IA: Iowa State University Press. 